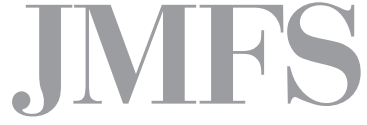

Journal of Management and Financial Sciences
Volume XI

Issue 31 (March 2018)

pp. 69-83

Warsaw School of Economics

Collegium of Management and Finance

Wioletta Mierzejewska

Collegium of Management and Finance

Warsaw School of Economics

\title{
Innovative Performance of Transnational Corporations
}

\begin{abstract}
The paper addresses innovative performance of transnational corporations (TNCs). Its goal is to find out whether innovation is typical of transnational corporations and what are its sources in TNCs. The goal has been attained by analysing the rankings of the world's most innovative companies. Three groups of transnational corporations featuring in these rankings have been distinguished: old giants, innovators with a past, and young players. Conclusions on the innovative performance of transnational corporations have been enriched with the analysis of sources of innovation in these companies, R\&D outlays included.
\end{abstract}

Key words: innovation, transnational corporations, research and development JEL Classification Codes: O30, F23, L14 


\section{Introduction}

Transnational corporations are important players in the global economy and drivers of internationalisation; they are also engines of globalisation. TNCs are active participants of economic processes that generate a considerable fraction of global GDP. It is estimated that they are indirectly involved in almost $80 \%$ of international trade and in many countries they are the main employers. Transnational corporations represent huge economic and political power, which not only helps them impact other enterprises but also governments and international organisations. International corporations directly impact entire societies, their standard of living and growth, including technological progress. At the same time, they are very much differentiated. TNCs can be found in high-tech industries as well as in traditional ones. Some are considerably engaged abroad with a large portion of their assets invested outside of their home countries, other focus on one market or region, being little engaged in foreign direct investment in other markets.

Due to the scale of their operations and economic power, transnational corporations are important actors worth researchers' attention, also when it comes to innovation. Moreover, apparently the scale of operations gives transnational corporations better access to knowledge and its transfer, while the assets they own make them best placed to generate and commercialise innovation. On the other hand, the subject-matter literature discusses innovation as a derivative of the creativity of an organisation, often associated with smaller companies or start-ups. Partial analyses suggest that creative organisational culture, flexible structures and creativity-friendly processes exert a positive impact upon innovative performance of companies. ${ }^{1}$ Most studies focus on innovation originating from small and medium-sized enterprises with a large creative potential. There are much fewer studies that would examine innovation in large transnational corporations.

Are transnational corporations innovative? Most of them are highly centralised and formalised giants with a mechanistic management style. Perhaps innovation in the early stages gave an impulse for their growth into what currently is a transnational corporation? The paper aims at answering the question if innovation as a reflection of creativity is an attribute of transnational corporations. If yes, what are its sources?

The paper outlines the substance and importance of transnational corporations to the global economy and discusses innovative performance of these operators plus identifies the main sources of their innovation. Discussion on innovative performance of transnational corporations is based on existing data taken mostly from a variety of rankings of the world's most innovative transnational corporations and from the UNCTAD ranking of transnational corporations. Thus, we will provide an initial overview of innovative performance of

1 See: A. Blomberg, T. Kallio, H. Pohjanpää, 2017. Antecedents of organizational creativity: Drivers, barriers or both? Journal of Innovation Management, vol. 5(1), pp.78-104. 
transnational corporations and identify innovation categories of transnational corporations to suggest directions for further research.

\section{Substance and importance of transnational corporations}

The subject-matter literature offers not only a series of definitions of transnational corporations but also a wide diversity of terms. Those most frequent combine the term "enterprise" or "corporation" with adjectives, such as international, multinational, global or transnational. ${ }^{2}$ Probably the most commonly used definition is the one proposed by the UN (UNCTAD), which asserts that a transnational corporation consists of a parent enterprise and its foreign affiliates. Parent enterprise is defined as an enterprise that controls assets of other entities in countries other than its home country, usually by owning a certain equity capital stake [Transnational corporations, UNCTAD, accessed 20.01.2018]. A transnational corporation is an enterprise, which, independently of its country of origin and type of ownership, brings together entities based in two or more countries bound by ownership or otherwise, so that one or more of them may be able to exercise a significant influence over the activities of others and, in particular, to share knowledge, resources and responsibilities with other entities. Transnational corporations operate under a system of decision making which permits coherent policies and a common strategy through one or more decision-making centres [Structure of TNCs, UNCTAD, accessed 20.01.2018].

Other definitions of transnational corporations additionally draw attention to aspects such as: operational and technological links as well as control-management relationships among entities within a transnational corporation [Jantoń-Drozdowska, 2006, pp. 155-169]; operations and investments all over the world often made through wholly or partly controlled companies [Ordonez, 2005, pp. 544-559]; engagement in foreign direct investment to control what its affiliates do in different countries [Dunning, 1993; Buckley and Casson, 2016]; aspects of a knowledge-based organisation [Kogut and Zander, 2003, pp. 516-529]; a possibility of creating optimal conditions to generate knowledge and attain maximum effects from its use [Nohria and Ghoshal, 1997] developing transborder knowledge transfer networks as effects of integration and coordination of geographically and organisationally dispersed efforts (Rosińska-Bukowska, 2009).

Diverse definitions result from changes in the way transnational corporations are organised, their operational scope and complex nature. One may observe how the definition of a transnational corporation evolved together with changes in the global economy. To start with, definitions of these complex structures highlighted ownership aspects and over time organisation-specific elements emerged from organisation, management and knowledge

2 See: M. Rosińska-Bukowska, 2009. Rola korporacji transnarodowych w procesach globalizacji. Kreowanie globalnej przestrzeni biznesowej. Script, available at: https://depot.ceon.pl/bitstream/handle/ [accessed 10.01.2018]. 
arrangement [Pakulska and Poniatowska-Jaksch, 2009, p. 85]. Nevertheless, we must note that the main attributes mentioned in various attempts of defining a transnational corporation include: operating in different geographical markets, substantial engagement of capital and assets in these markets, an ability to adjust to different time and space operating conditions, an inherent capability to coordinate and integrate scattered and diversified activities and global efficiency.

Irrespective of how we define these entities, they are meaningful actors of the global economy. Their role in the world's economy links with their capital, technological, and intellectual resources, that is with their production capacity. UNCTAD regularly produces the ranking of the largest transnational corporations by their foreign assets (Tab. 1).

Table 1. Ten largest TNCs according to UNCTAD in 2016

\begin{tabular}{|c|l|l|r|r|c|c|c|c|}
\hline \multirow{2}{*}{ No. } & \multirow{2}{*}{ Corporation } & \multirow{2}{*}{ Country of origin } & \multicolumn{2}{c|}{ Assets } & \multicolumn{2}{c|}{ Revenue from sales } & \multicolumn{2}{c|}{ Employment } \\
\cline { 5 - 9 } & & & Abroad & Total & Abroad & Total & Abroad & Total \\
\hline 1 & Royal Dutch Shell plc & United Kingdom & 349720 & 411275 & 152018 & 233591 & 67000 & 92000 \\
\hline 2 & Toyota Motor Corporation & Japan & 303678 & 435958 & 173529 & 254753 & 148941 & 348877 \\
\hline 3 & BP plc & United Kingdom & 235124 & 263316 & 140683 & 183008 & 43598 & 74500 \\
\hline 4 & Total SA & France & 233217 & 243468 & 110255 & 141526 & 70496 & 102168 \\
\hline 5 & Anheuser-Busch InBev & Belgium & 208012 & 258381 & 39507 & 45517 & 163177 & 206633 \\
\hline 6 & Volkswagen Group & Germany & 197254 & 431888 & 192093 & 240366 & 346715 & 626715 \\
\hline 7 & Chevron Corporation & USA & 189116 & 260078 & 54160 & 110484 & 28704 & 55200 \\
\hline 8 & General Electric Co & USA & 178525 & 365183 & 70352 & 123692 & 191000 & 295000 \\
\hline 9 & Exxon Mobil Corporation & USA & 165969 & 330314 & 121881 & 218608 & 35725 & 71100 \\
\hline 10 & Softbank Corp & Japan & 145611 & 220296 & 45324 & 82166 & 42032 & 63591 \\
\hline
\end{tabular}

Source: World Investment Report 2017. Investment and the Digital Economy, UNCTAD 2017.

Entities in the UNCTAD ranking are the world's largest transnational corporations, acting at an enormous scale with huge economic and political power generating substantial revenue from sales. Top ten of the largest transnational corporations include many operators from traditional industries and very few from modern technology sectors. That is caused mainly by the methodology applied for the ranking, which is based on their foreign assets. For obvious reasons the fuel sector attracts foreign investment, hence the domination of its entities in the top ten of the ranking.

Many analyses use the UNCTAD ranking as a good evidence of the importance of transnational corporations in the modern economy. Arguments in support of TNCs' importance in the economy can be found, inter alia, in the writings by W. Karaszewski [2004], J. Dunning [2007, pp. 177-199], M. Gasz [2012], S. Sala [2005, pp. 33-44], J. Kapler [1997, pp.195-211], A. Zorska [2007], M. Jaworek and M. Kuzel [205, pp.55-70], as well as other researchers. T. Pakulska and M. Poniatowska-Jaksch [2009, pp. 35-44] stress that the role of transnational corporations in the contemporary economy is usually assessed from the point of view of the four following elements: their economic potential, political importance, share in generating 
international trade, as well as scientific and research potential and technological progress. Surveys point to parameters such as employment (more than $82 \mathrm{~m}$ workers in foreign affiliates in 2016), revenue (USD 37, 570 bn generated by foreign affiliates in 2016), operations on the market of mergers and acquisitions (exceeding USD 869 bn in 2016) [World Investment Report 2017. Investment and the Digital Economy. UNCTAD, 2017] or their importance for international trade (ca. $80 \%$ takes place through or is linked with the value chains of transnational corporations $\left.{ }^{3}\right)$.

In conclusion, we need to note that transnational corporations are involved in globalisation and generate it at the same time, which is why their role in the growth of the world's economy is substantial [Sarbu, 2015, pp. 324-331]. Revenues they generate, the value of their market capitalization, R\&D potential, as well as the ability to coordinate and integrate dispersed assets, operations and entities should translate into transnational corporations' innovation skills.

\section{Innovative performance of transnational corporations}

Innovativeness is a feature exhibited by enterprises who are involved in innovation efforts, introduce or improve products, processes or management methods. It is reflected in innovation activities and the maturity of enterprises [Romanowska, 2016, pp. 29-35], as well as in their capability to generate, adapt and implement technological, organisational, and management innovation [Krasnicka, Głód and Wronka-Pospiech, 2016, pp. 132-142]. Models of innovation processes may differ significantly across enterprises and they evolve with the advancement of technological progress, globalisation, and changing customer requirements [Sopińska and Mierzejewska, 2017]. Due to changes emerging in the business environment, innovation is currently perceived as one of crucial management goals being, however, a wide category that is hard to quantify, although attempts have been made in the literature to develop its universal measure [Łukasik, 2017, pp. 416-423; Romanowska, 2017, pp. 107-122].

The paper uses a simplified method to assess innovative performance of transnational corporations. It is based on the analysis of existing data and on the investigation of a series of different rankings. First, we analysed the rankings published by UNCTAD for transnational corporations and then the rankings of the world's most innovative companies (not necessarily transnational corporations) published by magazines (Forbes, Fast Company), consulting companies (BCG, PwC), and a technical university (MIT).

UNCTAD studies unambiguously demonstrate that in the ranking of one hundred largest transnational corporations (by the value of their foreign assets) there is an increasing number of the so-called tech MNEs and telecom MNEs, that is corporations representing IT and technological industries. Between 2010 and 2015 their population doubled and currently it includes companies such as Apple, Hon Hai, IBM, Microsoft, Samsung, Sony and others. Even though

3 Press Release, UNCTAD, http://unctad.org/ (accessed 10.01.2018). 
only 19 out of one hundred largest transnational corporations are classified as tech and telecom $M N E s$, their share in the market value of all the corporations covered by the study is $32 \%$.

On top of that, UNCTAD (World Investment Report 2017] publishes a ranking of the top one hundred ICT transnational corporations (tech and telecom MNEs) and a ranking of the top 100 digital transnational corporations (digital MNEs). The ranking position depends on revenues from sales. Digital MNEs are those whose core of the business model is focused on the Internet. ICT corporations are entities that facilitate access to the Internet to individuals and to other businesses (hardware and software manufacturers, telecoms). Both rankings include very innovative companies, such as Apple, Samsung, Microsoft, Tencent, Qualcom, Netflix, Amazon, Alibaba, Salesforce, NetApp, Alphabet, Facebook, and many others. Innovation advancement of these corporations is undoubtedly linked with the industry that they represent, for which innovation is decisive in generating value and building up the competitive advantage (see Tab. 2).

Table 2. The largest transnational corporations in the ICT and digital sector in the UNCTAD ranking by sales in 2015

\begin{tabular}{|l|l|c|l|l|c|}
\hline \multicolumn{1}{|c|}{ Name } & \multicolumn{1}{|c|}{ Business area } & $\begin{array}{c}\text { Sales (in millions } \\
\text { of USD) }\end{array}$ & \multicolumn{1}{c|}{ Name } & \multicolumn{1}{c|}{ Business area } & $\begin{array}{c}\text { Sales (in millions } \\
\text { of USD) }\end{array}$ \\
\hline Amazon & Software and Internet & 107,006 & Apple & Computers and electronics & 215,639 \\
\hline Alphabet & Internet and software & 74,989 & Samsung & Electronics & 171,126 \\
\hline Comcast & Digital media & 74,510 & AT\&T & Telecommunications & 146,801 \\
\hline Time Warner & Digital media & 28,118 & $\begin{array}{l}\text { Hon Hai Precision } \\
\text { Industry }\end{array}$ & IT components & 135,996 \\
\hline $\begin{array}{l}\text { 21st Century } \\
\text { Fox }\end{array}$ & Digital media & 27,326 & $\begin{array}{l}\text { Nippon Telegraph } \\
\text { and Telephone }\end{array}$ & Telecommunications & 102,468 \\
\hline Liberty Global & Digital media & 18,280 & Microsoft & Software and Internet & 85,320 \\
\hline Facebook & $\begin{array}{l}\text { Communications and } \\
\text { Social media }\end{array}$ & 17,928 & IBM & Electronics, software & 81,741 \\
\hline Sky & Digital media & 16,138 & Softbank Group & Telecommunications & 81,271 \\
\hline Tencent & Games & 15,846 & Deutsche Telekom & Telecommunications & 75,368 \\
\hline Alibaba & Retail, Internet & 15,639 & Sony & IT equipment & 71,968 \\
\hline
\end{tabular}

Source: Technical Annex: The top 100 digital MNEs, WIR 2017 Chapter IV, UNCTAD 2017.

Unfortunately, UNCTAD surveys do not categorise transnational corporations based on their innovative performance plus the two above rankings focus exclusively on ICT and Internet industries, while innovations emerge in different sectors of the economy. UNCTAD surveys show financial performance and the efficiency with which these corporations use their assets. Apparently, the bigger the Internet content in transnational corporations' business model, the higher their international sales with limited engagement of assets abroad. For TNCs acting as Internet platforms, the index is the highest and reaches 2.6. In their case, little engagement of assets abroad translates into substantial international sales. 


\begin{tabular}{|c|c|c|c|c|c|c|c|c|c|c|c|}
\hline 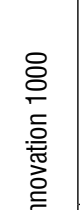 & 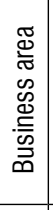 & 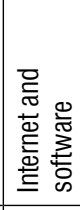 & 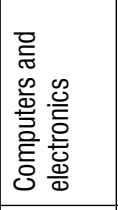 & 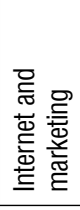 & 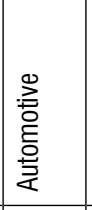 & 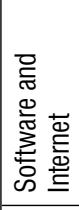 & 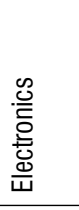 & 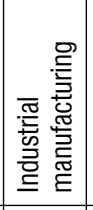 & 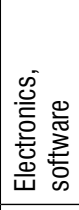 & 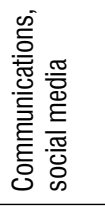 & 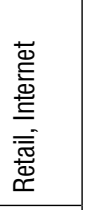 \\
\hline $\begin{array}{l}\ddot{0} \\
\text { 은 } \\
\dot{U} \\
\vdots \\
0\end{array}$ & 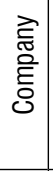 & 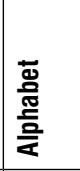 & $\frac{0}{\frac{2}{2}}$ & 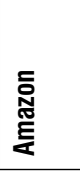 & 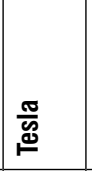 & 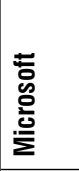 & 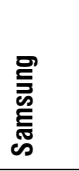 & 岕 & $\underline{\underline{\underline{\varphi}}}$ & 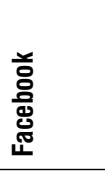 & 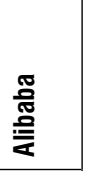 \\
\hline 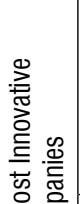 & 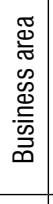 & 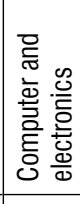 & 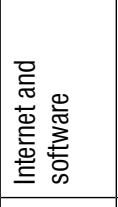 & 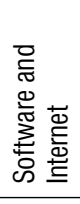 & 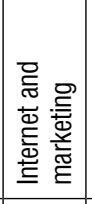 & 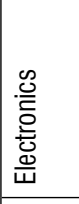 & 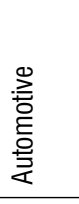 & 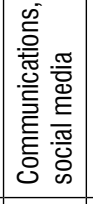 & 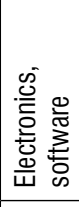 & 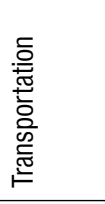 & 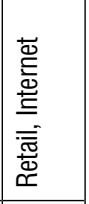 \\
\hline نூ & $\begin{array}{l}\text { స్ } \\
\text { ह్ } \\
\text { ठ․ }\end{array}$ & $\frac{0}{\frac{2}{2}}$ & 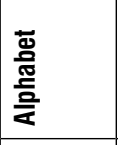 & 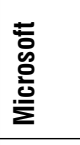 & 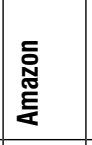 & 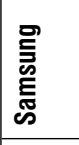 & 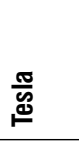 & 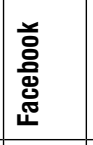 & $\underline{\underline{\underline{m}}}$ & 离 & 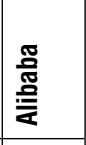 \\
\hline 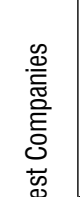 & 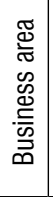 & 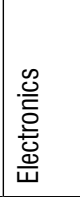 & 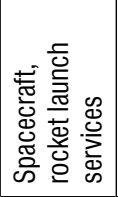 & 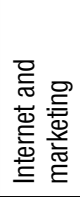 & 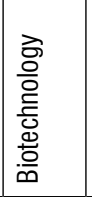 & 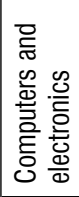 & 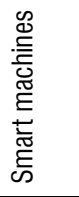 & 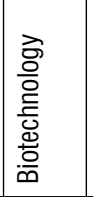 & 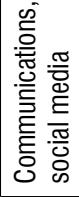 & 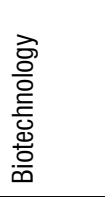 & 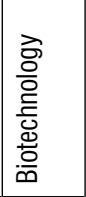 \\
\hline 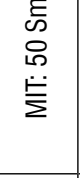 & 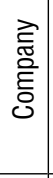 & 㗁 & 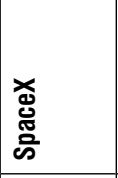 & 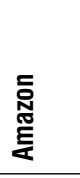 & 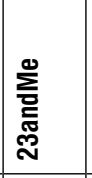 & 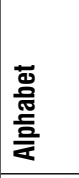 & 晜 & 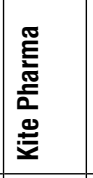 & 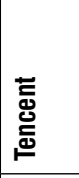 & 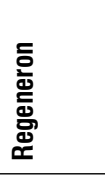 & 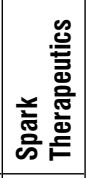 \\
\hline 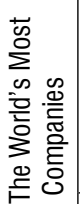 & 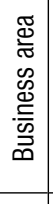 & 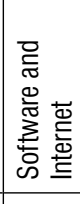 & 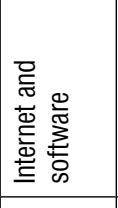 & 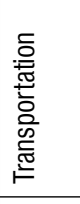 & 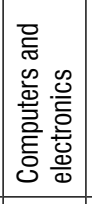 & 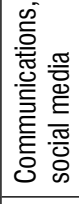 & 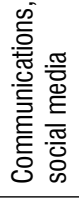 & 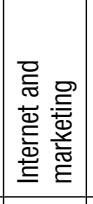 & 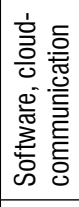 & 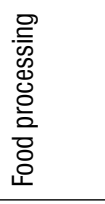 & 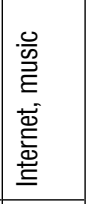 \\
\hline 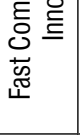 & 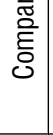 & 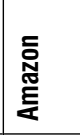 & 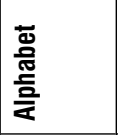 & ळ & $\frac{0}{\frac{0}{2}}$ & 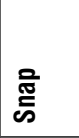 & 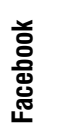 & 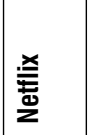 & 送 & 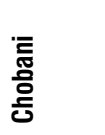 & 言 \\
\hline 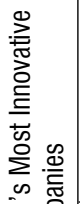 & 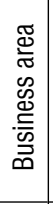 & 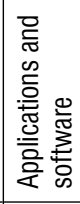 & 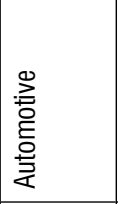 & 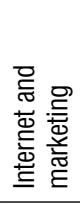 & 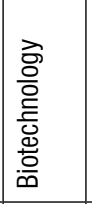 & 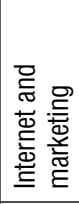 & 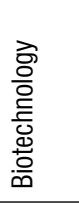 & 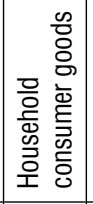 & 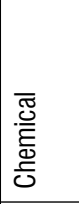 & 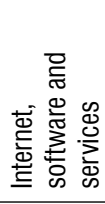 & 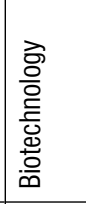 \\
\hline 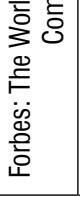 & 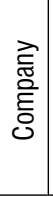 & 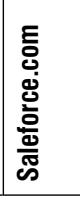 & 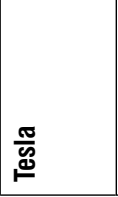 & 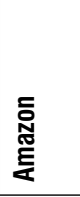 & 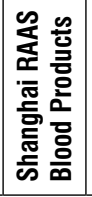 & 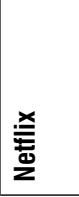 & 紊 & 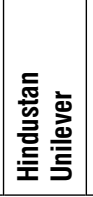 & 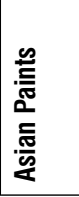 & 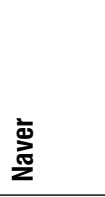 & 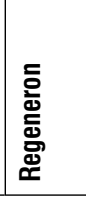 \\
\hline
\end{tabular}


The ranking published by UNCTAD is clearly focused on transnational corporations. However, it does not inform directly how innovative they are, which is why in order to draw conclusions, we also used other rankings identifying the most innovative global companies, which do not distinguish transnational corporations. Table 3 presents the top ten enterprises in the ranking of the world's most innovative companies.

Although not all the companies in Table 3 can be considered transnational corporations, TNCs have dominated most rankings (only the MIT ranking of 50 Smartest Companies is dominated by companies doing business at a more limited scale). Unfortunately, the rankings present a slightly divergent picture. Each of them features slightly different companies though there is a group that can be found in almost all of them. That is mostly due to the ranking methodology. Most rankings are questionnaire-based and respondents are asked to name the companies which they consider the world's most innovative businesses. This is the methodology applied, inter alia, in the $\mathrm{PwC}$ ranking, where the world's most innovative companies are named by respondents-innovation leaders (Ranking Global Innovation 1000). The BCG ranking is also largely based on opinions of managers, who are asked to identify the company they consider the world's most innovative business but it also takes account of financial indicators (Ranking of the Most Innovative Companies). Fast Company magazine has got a similar approach to the identification of the world's most innovative companies. Their list includes the companies which, according to experts, dazzle with new ideas and influence our contemporary market (Ranking of the World's Most Innovative Companies).

In Forbes, in the ranking of the world's 100 most innovative companies, businesses are not ranked by their earlier accomplishments but by the so-called innovation premium, which helps identify enterprises which are innovative now and are capable to be innovative in the future. Innovation premium is the difference between their market capitalization and the net present value of cash flows from existing businesses, given by equity investors on the educated hunch that the company will continue to come up with profitable new growth [Dyer and Gregersen, accessed 20.01.2018]. The famous technical university MIT has a different way to identify innovative companies. In its ranking it includes companies who are technologically innovative and have an effective business model (Ranking of the 50 Smartest Companies).

Despite some divergences resulting from the adopted methodological assumptions, we can see that some enterprises, such as Amazon, Alphabet, Apple or Facebook are coming back in several rankings, which only confirms transnational corporations' ability to be innovative. Nevertheless, we need to observe that TNCs featuring in the rankings of the world's most innovative companies represent very different profiles. In general, we may identify three groups of TNCs in innovation rankings. The first group includes "old giants" trying to survive in new technology industries (e.g., GE, IBM or Samsung). The group, however, is not uniform and we may find there entities which in their early years were innovative and innovation helped them grow and become transnational corporations (e.g., IBM, GE) hand in hand with "old giants", who at the start of their activities were not innovative at all. Innovation came with time, perhaps out of necessity to survive in the evolving market and fight for a solid global 
competitive position or as a result of the impact exerted by the government of the home country (e.g., Samsung started as a wholesaler of fish, fruit and vegetables).

The second group of innovative transnational corporations comprises "innovators with a past", that is corporations which emerged from the waves of computer revolution of the 1970 s and 1980s. They are usually innovative businesses involved in the development of Personal Computers, terminals, and software (e.g., Microsoft, Apple). They are also beneficiaries of globalisation and liberalisation of global trade, as well as rapid dissemination of new IT technologies. Nowadays, they enter new fields and continue to master their core business (e.g., Apple and energy generation).

The third group of TNCs included in the rankings of the most innovative companies is composed of relatively "young players", i.e., entities established in the late $20^{\text {th }}$ or early $21^{\text {st }}$ centuries. These TNCs have been fuelled by the Internet-related innovations, which have helped small businesses to become huge and powerful global players over a relatively short time. This group, even though little uniform, has dominated the tops of innovation rankings. Beside businesses established in the 1990s (such as Amazon, Netflix, Alibaba or Saleforce) it includes very young businesses, such as Facebook, Chobani or Uber, which despite being in the market for only several years can already be considered transnational corporations. This is where the power of innovation becomes even more obvious in making them grow and transform into global corporations. Both groups benefit from globalisation, intense development of ICT, virtualisation of business processes, and moving them onto the Internet.

Summing up, we can positively answer the question about innovative performance of transnational corporations. In spite of the scale of their business, TNCs are considered top global innovators. For some of them, innovation results from the industry, in which they operate (Internet platforms, software producers, etc.) and is a must for building a competitive advantage; often it was also an impulse for their global growth. For other TNCs, the need for innovation emerged over the course of their development and is linked with the evolution in the scope of their business. However, lists of the world's most innovative companies are dominated by corporations who started and developed dynamically as innovative businesses. The question is how long they will be able to generate innovation as some corporations, which in the early years featured in innovation rankings can no longer be found amongst the world's most innovative companies (e.g. Siemens, Dell, DuPont or Philips).

\section{Sources of innovation in transnational corporations}

Since we can consider transnational corporations innovative businesses, a question must be asked about the sources of these innovations. The subject-matter literature identifies two sources: internal and external. The main sources of innovation in TNCs come from their own research and development activities and all sorts of cooperation arrangements aimed at the transfer of technology, knowledge or skills [Nowakowski, 2005, pp. 388-293]. In the first model 
of generating innovation, R\&D outlays are critical, while the second model needs stronger cooperation links because it is based on the so called open innovation. Some researchers distinguish the third model of generating innovation in TNCs, an engineering innovation model, which needs to effectively combine product development and engineering processes. The model calls for the engagement of suppliers, advanced logistics and emerges in countries of strong engineering culture [Herstatt et al., 2006, p. 311].

According to UNCTAD estimates, in the early $21^{\text {st }}$ century transnational corporations were responsible for over half of total R\&D expenditure and $69 \%$ of global R\&D corporate expenditure. Besides, R\&D budgets of some corporations (e.g. Ford, Pfizer, Siemens, Toyota, GM) were higher than some national R\&D budgets [Pakulska and Poniatowska-Jaksch, 2009, p. 41]. The PwC ranking of the top R\&D spenders examines enterprises who spend the most on R\&D. Enterprises included in the ranking account for over $40 \%$ of global R\&D expenditure. All the companies spent more than USD 702 bn on R\&D in 2017 (3.2\% more than in the preceding year). Most innovation spending comes from: electronics, health care, software and the Internet, and automotive industries. The top ten enterprises from the ranking are presented in Table 5.

Table 5. Top R\&D spenders according to PwC

\begin{tabular}{|l|c|}
\hline \multicolumn{1}{|c|}{ Company } & R\&D spending (in billions of USD) \\
\hline Amazon & 16.1 \\
\hline Alphabet & 13.9 \\
\hline Intel Corporation & 12.7 \\
\hline Samsung Electronics & 12.7 \\
\hline Volkswagen (VW) & 12.1 \\
\hline Microsoft Corporation & 12 \\
\hline Roche holding & 11.4 \\
\hline Merck & 10.1 \\
\hline Apple & 10 \\
\hline Novartis & 9.6 \\
\hline
\end{tabular}

Source: Ranking Top R\&D Spenders 2017, PwC.

Research and development are significant sources of innovation for TNCs. Nevertheless, one may realise that among top ten $\mathrm{R} \& \mathrm{D}$ spenders there are some who are not considered the most innovative. The comparison of the above ranking with the list of the world's most innovative companies reveals some divergences. Some names repeat but the businesses such as VW, Intel, Merck, Roche or Novartis cannot be found in the top 10 of any world's most innovative companies ranking. Neither can they be found in the top 100 world's most innovative companies of the Forbes ranking with only Intel featuring in the latest BCG ranking of the most innovative companies. On top of that, if we compare the financial performance of companies from the list of the most innovative companies and from the ranking of the top $R \& D$ spenders, the first ones are doing much better. The most innovative companies from the $\mathrm{PwC}$ 
ranking report higher average sales, EBITDA, and market growth. It means there is no simple relationship between $\mathrm{R} \& \mathrm{D}$ expenditure and innovation. You may spend but not necessarily be innovative and the global market provides examples of a reversed relationship when companies are innovative without excess $R \& D$ spending. Thus, it is not only the corporate budget that counts but, predominantly, the efficiency with which it is spent.

$\mathrm{PwC}$ studies provide evidence that how one spends $\mathrm{R} \& \mathrm{D}$ budget is of paramount importance, not only the budget size [PwC, 2017]. According to the Institute of Innovative Economy [Sznyk and Karasek, 2015]. there is no innovation without R\&D expenditure, human capital and incentives for business. The roles of human capital and corporate internal entrepreneurial spirit need to be highlighted. Perhaps this is what companies such as VW, Merck or Novartis are missing. Additionally, they have overlooked the right moment to invest in sectoral innovations, which are slowly becoming standard. These companies must catch up with market leaders and in many instances they want to do it through high R\&D spending. In their case, R\&D expenditure alone does not prejudge about generated outcomes as what is crucial is the strategy and the right moment of its implementation. Historically, one may enumerate many entities which made strategic mistakes by overlooking the development of new technologies (e.g. Nokia).

When trying to explain the discrepancy between R\&D outlays and the position in the innovation ranking, it is worth quoting T. Amabile's analyses [Amabile, 1997, p. 39]. The researcher argues that innovation is a derivative of creativity of employees and teams thereof, as well as assets of an organisation, its management practices and overall innovation mindset in the enterprise. Thus, we need to note that perhaps companies whose $R \& D$ expenditure is substantial lack soft attributes decisive for creativity of an organisation and, as a result, an ability to generate innovation.

Nevertheless, $R \& D$ spending remains the primary source of innovation in transnational corporations. Moreover, some authors believe that innovation can be generated only in large enterprises or organisations, which assist their employees in developing ideas by creating conditions conducive to the sharing of knowledge and experimenting and ensure material support [Arak, 2014]. In his book W. Dyduch asserts that organisational creativity (and innovation as its next stage) results from interactions among employees, which, if permitted by the organisation, enhance the probability of new ideas being created. Take for instance the $3 \mathrm{M}$ corporation, which allows its employees to spend $15 \%$ of their working time on generating new ideas not related with their everyday work [Dyduch, 2013, p. 58]. It seems that only large organisations can afford to adopt such solutions.

$\mathrm{R} \& \mathrm{D}$ effort can be organised differently in transnational corporations. In extreme cases it may be centralised and delivered top-down or be geographically dispersed. Market trends show that TNCs increasingly more often avail themselves of this second option of organising their R\&D. TNCs are becoming hunters for strategic resources, which are supposed to facilitate the implementation of innovation for them [Markusen, 1995, pp. 169-189; Manea and Pearce, 2004, pp. 7-28]. Their innovative performance is also influenced by possibilities of accumulating and developing technologies in different geographic regions [Cantwell, 1989]. 
On top of that, they continuously reorganise themselves to avoid ending up as petrified organisational structures. For instance, Tesla, a typical automotive OEM, is organised around small, skilful teams, which ensures higher innovation [BCG, 2018]. On the other hand, innovative Apple is a rather hierarchical and centralised corporation, which ensures highly integrated and coherent operations. However, in such an organisational pattern a lot depends on the top managing person, her/his ideas and development vision [Leśniak-Moczuk, 2016, pp. 33-40].

Big corporate budgets enable TNCs to benefit from a variety of sources of innovation. Availability of diverse resources, effects of technology dissemination and other factors may suggest their higher ability to generate innovation. Many transnational corporations acquire innovation by acquiring start-ups. Companies such as, inter alia, Alphabet, Facebook or Amazon are very active in this field. These corporations have huge financial resources and can acquire small innovative entities. Looking at the rankings of the world's top innovative companies, the strategy seems rather effective. It also partly explains the earlier presented scale of mergers and acquisitions of transnational corporations.

Innovation in TNCs may also come from their being better placed to enter a variety of alliances with their competitors, contractual relations with research institutions or suppliers, and engaging customers in generating innovation. One of the directions to promote innovation development in TNCs is open innovation and innovation implemented in cooperation with a series of entities using combined own and external resources (including knowledge), which may produce considerable synergy effects and translate into more innovation.

No doubt TNCs have a lot of potential to generate and commercialise innovation. They are considered best placed to compete within the framework of the knowledge-based economy as they have accumulated their own knowledge ( $R \& D$ research) and may benefit from the knowledge accumulated by their partners across the world; they can also easily and smoothly disseminate the acquired knowledge through their internal distribution channels [Rosińska-Bukowska, 2009]. Sources of TNCs innovation are anchored in their inner capabilities but also in their budgets, which help acquire innovative start-ups and result from the specificity of their modus operandi in many geographical locations, which promotes knowledge and technology transfer. One of the features of transnational corporations is their ability to integrate knowledge coming from different sources and cultures, which enhances the innovative potential.

\section{Summary}

The paper focuses on innovative performance of transnational corporations, which are interesting subjects to examine because of their importance to the global economy. Their size measured with revenue, assets or employment has attracted the interest of researchers but also media and governments. In the age of the digital economy, the question about the extent to which these huge entities are innovative and whether they operate only in traditional sectors or also in high-tech ones remains valid and vital. Studies show that TNCs willingly 
invest in fast growing and hi-tech sectors [Kapler, 1997, pp. 195-211]. J. Kapler stresses that perhaps TNCs are more successful in hi-tech sectors than national corporations operating only in one country, because they can easily transfer knowledge and technology.

Interestingly, innovation of transnational corporations is not linked with the size of their foreign assets. TNCs whose value of foreign assets is higher often originate from traditional less innovative industries, e.g., fuel or processing industries. Development of new technologies has contributed to the development of TNCs, which despite relatively little engagement of foreign assets exhibit high levels of innovation.

The paper illustrates innovation in transnational corporations with a series of rankings. Unfortunately, such an analysis has got its limitations, since the rankings are hard to compare due to their different methodologies, the unrepresentative research sample and a lack of possibility of using statistical analysis methods, which identify significant relationships.

However, this analysis helped produce an initial overview of the situation. It turns out that transnational corporations not only have an innovative potential (understood as access to knowledge, human resources, technology and finance) but are among the world's most innovative companies, which is reflected in their positions in the above described rankings, which, however, comprise very divergent entities. The paper identifies three main categories of innovative transnational corporations ("old giants", "innovators with a past" and "young players"), based on their track record. Their sources of innovation rest with their own R\&D activities, whose budgets sometimes exceed state budgets of smaller countries, but also with capabilities to acquire innovative businesses and enter cooperation alliances across the world, providing them with better access to new knowledge, ideas and technologies.

As demonstrated in the paper, innovation of transnational corporations is an interesting research subject that merits further investigation. In subsequent studies we would like to confirm the thesis on innovative performance of transnational corporations and its sources on a representative research sample and using primary data. Interesting directions of further studies include comparisons within and across industries, as well as between the geographic origin of transnational corporations or their engagement abroad. Another question to be further explored includes conditions favouring innovation in transnational corporations, success factors and ramifications. We may conclude that due to the importance of TNCs to the global economy we should investigate each and every aspect of their operations, including their ability to generate and implement innovations.

\section{References}

1. Amabile T.M., 1997. Motivating creativity in organizations: on doing what you love and loving what you do. California Management Review, vol. 40, no. 1.

2. Arak P., August 2014. Nie łudźmy się, mała firma nie stworzy innowacj. Available at: https:// www.obserwatorfinansowy.pl/ [Accessed 10.01.2018]. 
3. Blomberg A., Kallio T., Pohjanpää H., 2017. Antecedents of organizational creativity: Drivers, barriers or both? Journal of Innovation Management, vol. 5(1).

4. BCG January 2018. The most innovative companies 2018. Innovators go all in on digital. https:// www.bcg.com/ [accessed 20.01.2018].

5. Buckley P.J., Casson M., 2016. The future of the multinational enterprise. McMillan Press.

6. Cantwell J., 1989. Technological innovation and multinational corporations. Blackwell.

7. Dunning J., 1993. Multinational enterprises and the global economy., Reading, MA: Addison Wesley. In: A. Zorska, 2007. Korporacje transnarodowe. Przemiany, oddziaływania, wyzwania. Warszawa: PWE.

8. Dunning J., 2007. Some macro-data on the regionalisation/globalisation debate: a comment on the Rugman/Werbke analysis. Journal on the International Business Studies, vol. 38.

9. Dunning J., 1993. The theory of transnational corporations. vol. 1. Taylor \& Francis.

10. Dunning J., Lundan S., 2008. Multinational enterprises and the global economy. Edward Elgar Publishing.

11. Dyduch W. 2013. Twórcza strategia organizacji. Katowice.

12. Dyer J., Gregersen H., How We Rank the Most Innovative Companies 2017. Forbes. Available at: https://www.forbes.com/ [Accessed 20.01.2018].

13. Gasz M., 2012. Znaczenie korporacji transnarodowych w gospodarce światowej. Ekonomia i Prawo, vol. 11.4 .

14. Herstatt C. et al., 2012. Management of technology and innovation in Japan, Springer Verlag, Berlin-Heidelberg 2006, p. 311. In: J. Rymarczyk, Biznes międzynarodowy. Warszawa: PWE.

15. Jantoń-Drozdowska E., 2006. Korporacje transnarodowe w procesie globalizacji. Ruch prawniczy, ekonomiczny i socjologiczny, vol. 2.

16. Jaworek M., Kuzel M., 2015. Transnational corporations in the world economy: Formation, development and present position. Copernican Journal of Finance and Accounting, vol. 4(1).

17. Kapler J.K., 1997. The theory of transnational firms: An empirical reassessment. International Review of Applied Economics, vol.11(2).

18. Karaszewski W., 2004. Największe korporacje transnarodowe świata i ich wpływ na wzrost gospodarczy. In: M. Hafler, W Karaszewski, Eds. Czynniki wzrostu gospodarczego. Toruń: Wydawnictwo Uniwersytetu Mikołaja Kopernika.

19. Kogut B., Zander U., 2003. Knowledge of the Firm and the Evolutionary Theory of Multinational Corporation. Journal of International Business Studies, vol. 34.

20. Kraśnicka T., Głód W., Wronka-Pospiech M., 2016. Innowacyjność polskich gazel biznesu. Studia Ekonomiczne. Zeszyty Naukowe UE w Katowicach, no. 270.

21. Leśniak-Moczuk A., 2016. Wizja, strategia, innowacyjność w firmie Apple. Finanse, Rynki Finansowe, Ubezpieczenia, no. 5(83), part 2.

22. Łukasik P., 2007. Analiza problemów pomiaru innowacyjności przedsiębiorstw. Nierówności Społeczne a Wzrost Gospodarczy, no. 52(4).

23. Manea J., Pearce R., 2004. Industrial restructuring in economies in transition and TNCs' investment motivations. Transnational Corporations, vol. 13.2. 
24. Markusen J., 1995. The boundaries of multinational enterprises and the theory of international trade. The Journal of Economic Perspectives, vol. 9.2.

25. Nohria N., Ghoshal S., 1997. The Differentiated Network. Organizing Multinational Corporations for Value Creation. San Francisco: Jossey-Bass Publishers.

26. Nowakowski M.K., Ed., 2005. Biznes międzynarodowy. Od internacjonalizacji do globalizacji. Warszawa: OW SGH.

27. Ordóñez P., 2005. Transnational corporations and strategic challenges, The Learning Organization, vol. 13(6).

28. Pakulska T., Poniatowska-Jaksch M., 2009. Korporacje transnarodowe a globalne pozyskiwanie zasobów. Warszawa: OW SGH.

29. $\mathrm{PwC}, 2017$. Reinventing innovation. Five findings to guide strategy through execution. Available at: https://www.pwc.com/ [Accessed 10.01.2018].

30. Romanowska M., 2016. Determinanty innowacyjności polskich przedsiębiorstw. Przegląd Organizacji, no. 2.

31. Romanowska M., 2017. Profil diagnostyczny jako narzędzie badania innowacyjności przedsiębiorstw. STUDIA OECONOMICA POSNANIENSIA, vol. 5, no. 9.

32. Rosińska-Bukowska M., 2009. Rola korporacji transnarodowych w procesach globalizacji Kreowanie globalnej przestrzeni biznesowej. Script. Available at: https://depot.ceon.pl/bitstream/ handle/ [Aaccessed 10.01.2018].

33. Sala S., 2005. Rozwój korporacji transnarodowych w gospodarce światowej. Przedsiębiorczość-Edukacja, vol. 1.

34. Sarbu M., 2015. Globalization and Foreign Direct Investments. Iasi: Alexandru Ioan Cuza University of Iasi, Centre for European Studies, CES Working Papers, vol. VII, iss. 2.

35. Sopińska A., Mierzejewska W., 2017. Otwarte innowacje produktowe realizowane przez przedsiębiorstwa działające $w$ Polsce. Podejście zasobowe. Warszawa: OW SGH.

36. Sznyk A., Karasek J., Eds., March 2015. Innowacje 2015. Available at: http://ingos.pl/public/, [accessed 10.01.2018].

37. UNCTAD, Press Release. Available at: http://unctad.org/ [Accessed 10.01.2018].

38. UNCTAD. Structure of TNCs. Available at: http://unctad.org/ [Accessed 20.01.2018].

39. UNCTAD, 2017a. Technical Annex: The top 100 digital MNEs, WIR 2017 Chapter IV. http:// unctad.org/ [Aaccessed 10.01.2018].

40. UNCTAD. Transnational corporations. http://unctad.org/en/Pages/DIAE/Transnationalcorporations- (TNC). aspx [Accessed 20.01.2018].

41. UNCTAD, 2017b. World Investment Report 2017. Investment and the Digital Economy. http:// unctad.org/ [accessed 10.01.2018].

42. Zorska A., 2007. Korporacje transnarodowe. Przemiany, oddziaływania, wyzwania. Warszawa: PWE. 
